\title{
Vascular invasion affects survival in early hepatocellular carcinoma
}

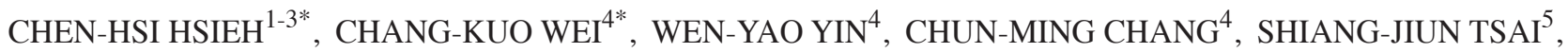 \\ LI-YING WANG ${ }^{6,7}$, WEN-YEN CHIOU ${ }^{5}$, MOON-SING LEE ${ }^{5,8}, \mathrm{HON}_{-Y I} \mathrm{LIN}^{5,8}$ and SHIH-KAI HUNG ${ }^{5,8}$ \\ ${ }^{1}$ Department of Radiation Oncology, Far Eastern Memorial Hospital, New Taipei City 220; \\ ${ }^{2}$ Department of Medicine; ${ }^{3}$ Institute of Traditional Medicine, School of Medicine, National Yang-Ming University, \\ Taipei City 112; Departments of ${ }^{4}$ General Surgery and ${ }^{5}$ Radiation Oncology, Buddhist Dalin Tzu Chi Hospital, \\ Dalin, Chiayi 62247; ${ }^{6}$ School and Graduate Institute of Physical Therapy, College of Medicine; \\ ${ }^{7}$ Physical Therapy Center, National Taiwan University Hospital, Taipei City 100; \\ ${ }^{8}$ School of Medicine, Tzu Chi University, Hualien 970, Taiwan, R.O.C.
}

Received August 12, 2014; Accepted September 12, 2014

DOI: $10.3892 / \mathrm{mco} .2014 .420$

\begin{abstract}
Hepatocellular carcinoma (HCC) is an aggressive malignant tumor with a high mortality rate. The optimal therapeutic choice for early $\mathrm{HCC}$ is surgical resection. However, the rate of intrahepatic recurrence is high. The objective of this study was to evaluate the effect of various factors on the survival of patients with early HCC. Between January 1st, 2006 and December 31st, 2013, a total of 89 patients who underwent surgery for HCC were retrospectively enrolled. The analysis was conducted using the Student's t-test, Chi-square test, Kaplan-Meier method and Cox proportional hazard regression model to assess potential confounding and predictive variables. The 3-year overall survival (OS) rate was 71\%. The 3-year OS rates in patients with and those without vascular invasion were 62.1 and $92.8 \%$, respectively $(\mathrm{P}<0.003)$. Based on the multivariate analysis, postoperative pathological vascular invasion (hazard ratio $=4.96 ; 95 \%$ confidence interval: $1.55-15.9)$ remained an independent predictor of adverse long-term outcome. Furthermore, vascular invasion was significantly associated with intrahepatic metastasis. These data emphasize the need for effective adjuvant therapy in selected high-risk patients with early HCC. Further studies are required to determine the optimal approach to further improving the prognosis of early HCC.
\end{abstract}

Correspondence to: Dr Shih-Kai Hung, Department of Radiation Oncology, Buddhist Dalin Tzu Chi General Hospital, 2 Ming Sheng Road, Dalin, Chiayi 62247, Taiwan, R.O.C.

E-mail: oncology158@yahoo.com.tw

*Contributed equally

Key words: hepatocellular carcinoma, surgery, vascular invasion

\section{Introduction}

Hepatocellular carcinoma (HCC) is an aggressive malignant tumor with a high mortality rate (1). Several modalities, including surgical resection, transcatheter arterial chemoembolization (TACE) and arterial infusion chemotherapy, percutaneous ethanol injection therapy, microwave coagulation therapy, radiotherapy and liver transplantation, have been used in the treatment of $\operatorname{HCC}(2,3)$. The optimal choice for patients with early HCC is surgery, as resection may be easily performed, with low mortality rates (4). However, the rate of intrahepatic recurrence is high (5). Poon et al (6) reported that $43 \%$ of the HCC patients developed recurrence following resection with intrahepatic metastasis or multicentric occurrence of a new tumor in the residual liver. Factors such as patient age, size and number of tumors, presence of a tumor capsule, vascular invasion, histological grade, pathological TNM stage and surgical resection margins, have all been demonstrated to affect recurrence postoperatively (7-9). The improved survival following hepatectomy for HCC has been mainly attributed to the prevention of recurrence. The aim of this study was to evaluate the results and prognostic factors in the surgical treatment of early HCC in the same institution.

\section{Materials and methods}

Ethics statement. The study protocol was approved by the Institutional Review Board of Buddhist Dalin Tzu Chi General Hospital (no. B10102004). The Institutional Review Board waived the requirement for written informed consent from the participants, as the data released from the hospital database were analyzed anonymously.

Patients. Between January 1st, 2006 and December 31st, 2013 , a total of 89 patients diagnosed with HCC, who underwent partial hepatectomy with complete resection of gross disease, were retrospectively enrolled. Intraoperative ultrasound was routinely used to determine the anatomical characteristics 
Table I. Characteristics of HCC patients.

\begin{tabular}{|c|c|c|}
\hline Variables & $\begin{array}{l}\text { No. of patients } \\
\qquad(\mathrm{n}=89)\end{array}$ & $\%$ \\
\hline \multicolumn{3}{|c|}{ Age (years) } \\
\hline$<60$ & 44 & 49.4 \\
\hline$\geq 60$ & 45 & 50.6 \\
\hline \multicolumn{3}{|l|}{ Gender } \\
\hline Male & 66 & 74.2 \\
\hline Female & 23 & 25.8 \\
\hline \multicolumn{3}{|l|}{ pT } \\
\hline pT1 & 39 & 43.8 \\
\hline pT2 & 50 & 56.2 \\
\hline \multicolumn{3}{|c|}{ Vascular invasion } \\
\hline No & 50 & 56.2 \\
\hline Yes & 39 & 43.8 \\
\hline \multicolumn{3}{|c|}{ Tumor size, mm } \\
\hline$<45$ & 64 & 71.9 \\
\hline$\geq 45$ & 25 & 28.1 \\
\hline \multicolumn{3}{|c|}{ Surgical margin } \\
\hline$(+)$ & 4 & 4.5 \\
\hline$(-)$ & 85 & 95.5 \\
\hline$\leq 1 \mathrm{~mm}$ & 14 & 15.7 \\
\hline$>1 \mathrm{~mm}$ & 75 & 84.3 \\
\hline \multicolumn{3}{|l|}{ Stage } \\
\hline I & 39 & 43.8 \\
\hline II & 50 & 56.2 \\
\hline \multicolumn{3}{|c|}{ BCLC stage } \\
\hline 0 & 20 & 22.5 \\
\hline A & 46 & 51.7 \\
\hline B & 23 & 25.8 \\
\hline
\end{tabular}

HCC, hepatocellular carcinoma; pT, pathological T stage; BCLC, Barcelona-Clinic Liver Cancer.

of the tumor and to evaluate the residual liver for additional tumors. All the patients were evaluated with a baseline history and physical examination, serum laboratory tests and a computed tomography or magnetic resonance imaging scan of the abdomen and pelvis.

Follow-up. The patients were followed up postoperatively with physical examinations, serial computed tomography scans, or ultrasonography and $\alpha$-fetoprotein levels at 3-6-month intervals (if the level was elevated preoperatively) for the first year and every 6 months thereafter. All the patients included in this analysis had a minimum 6 months of follow-up. Recurrence of HCC was identified by new lesions on imaging with characteristics typical of HCC or increasing $\alpha$-fetoprotein levels. Lesions that were not typical of HCC were confirmed by biopsy. The pathological specimens were reviewed for the following tumor characteristics: Number and size of tumors, tumor grade, vascular invasion and microscopic margins. A

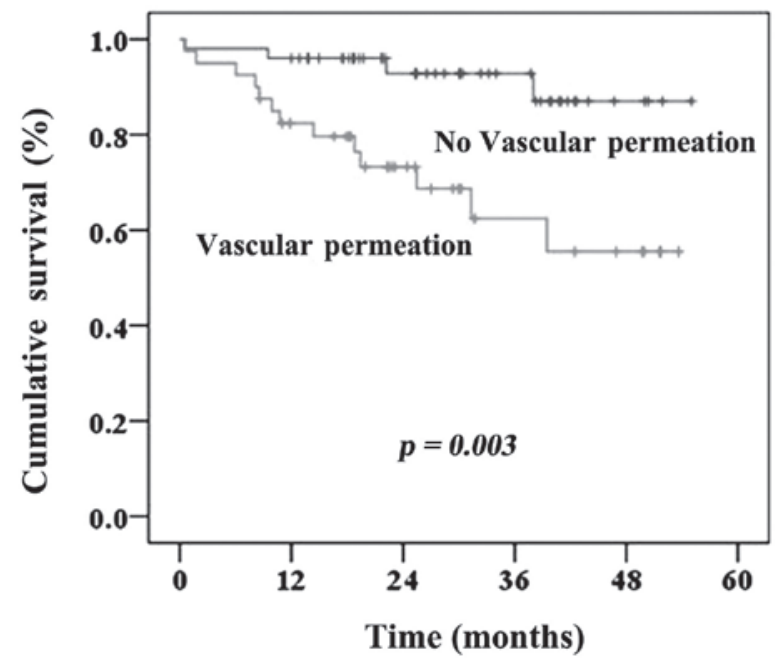

Figure 1. Three-year overall survival curve for all hepatocellular carcinoma patients according to the presence or absence of vascular invasion.

margin between 0 and $\leq 1 \mathrm{~mm}$ was considered to be a close margin. Positive margins indicated histological involvement. Pathological vascular invasion was defined as encroachment of cancer cells into blood vessels confirmed pathologically. Macrovascular involvement was defined as histological involvement of lobar or segmental branches of the portal or hepatic veins, or gross invasion of the right or left main branches of the portal or hepatic veins.

Statistical analysis. Patient demographics, tumor, surgical treatment and treatment characteristics were evaluated. The following variables were analyzed: Age, gender, Child-Pugh classification of cirrhosis, $\alpha$-fetoprotein level, hepatitis serological status and extent of liver resection. Comparisons between groups were performed with the Chi-square test for categorical variables and the Student's t-test for continuous variables. The Kaplan-Meier analysis was used and the results for subgroups of patients were compared with a log-rank test (SPSS software, version 17.0; SPSS, Inc., Chicago, IL, USA). All the variables that appeared to be significantly associated with survival $(\mathrm{P}<0.05)$ were entered into a Cox proportional hazards model to assess significant effects and adjust for multiple factors simultaneously. $\mathrm{P}<0.05$ was considered to indicate a statistically significant difference.

\section{Results}

Patient characteristics. The patient characteristics are summarized in Table I. The majority of the patients $(74.2 \%)$ had Barcelona-Clinic Liver Cancer stage 0 and A disease by clinical and laboratory evaluation. Pathological vascular invasion was identified in 39 patients $(43.8 \%)$. A total of 50 patients had liver cirrhosis and a total of 36,37, 9 and 7 patients had hepatitis $\mathrm{B}$ virus (HBV) infection, hepatitis $\mathrm{C}$ virus (HCV) infection, non-HBV/HCV hepatitis and concurrent $\mathrm{HBV} / \mathrm{HCV}$ hepatitis, respectively.

Follow-up. The median follow-up time was 27.2 months. The median time-to-recurrence was $8.8 \pm 4.5$ months 
Table II. Three-year clinical outcomes according to prognostic factors.

\begin{tabular}{|c|c|c|c|c|c|c|c|c|c|c|c|c|}
\hline Factors & $\begin{array}{c}\text { Overall } \\
\text { survival } \\
(\%)\end{array}$ & $\mathrm{P}$ & $\begin{array}{l}\text { Disease- } \\
\text { free } \\
\text { survival } \\
(\%)\end{array}$ & $P$ & $\begin{array}{c}\text { Disease- } \\
\text { specific } \\
\text { survival } \\
(\%)\end{array}$ & $\mathrm{P}$ & $\begin{array}{c}\text { Local } \\
\text { control } \\
(\%)\end{array}$ & $\mathrm{P}$ & $\begin{array}{c}\text { Intra- } \\
\text { hepatic } \\
\text { control } \\
(\%)\end{array}$ & $\mathrm{P}$ & $\begin{array}{c}\text { Distant } \\
\text { metastasis- } \\
\text { free } \\
\text { rate }(\%)\end{array}$ & $\mathrm{P}$ \\
\hline \multicolumn{13}{|l|}{ Age (years) } \\
\hline$<60 / \geq 60$ & $78.1 / 80.9$ & 0.13 & $62.4 / 40.5$ & 0.67 & $82.0 / 80.9$ & 0.42 & $91.7 / 86.6$ & 0.36 & $72.7 / 46.4$ & 0.33 & $97.6 / 97.8$ & 0.93 \\
\hline \multicolumn{13}{|l|}{ Gender } \\
\hline $\mathrm{M} / \mathrm{F}$ & $75.6 / 90.0$ & 0.13 & $51.4 / 50.6$ & 0.44 & $78.1 / 90.0$ & 0.25 & $84.9 / 100.0$ & 0.05 & $62.7 / 50.6$ & 0.63 & $100.0 / 96.8$ & 0.38 \\
\hline \multicolumn{13}{|l|}{$\mathrm{pT}$} \\
\hline $\mathrm{pT} 1 / \mathrm{pT} 2$ & $90.6 / 71.1$ & 0.08 & $61.1 / 43.0$ & 0.09 & $93.0 / 72.5$ & 0.02 & $92.1 / 86.3$ & 0.38 & $69.0 / 50.5$ & 0.05 & $100.0 / 95.8$ & 0.20 \\
\hline \multicolumn{13}{|l|}{ VI } \\
\hline No/yes & $92.8 / 62.1$ & $<0.003^{\mathrm{a}}$ & $60.9 / 37.9$ & $<0.005^{\mathrm{a}}$ & $94.7 / 63.7$ & $<0.001^{\mathrm{a}}$ & $91.5 / 85.6$ & 0.40 & $66.8 / 47.8$ & $0.008^{\mathrm{a}}$ & $100.0 / 94.5$ & 0.09 \\
\hline \multicolumn{13}{|c|}{ Tumor size (mm) } \\
\hline$<50 / \geq 50$ & $81.8 / 72.1$ & 0.24 & $52.3 / 46.0$ & 0.34 & $84.3 / 72.1$ & 0.08 & $88.9 / 88.9$ & 0.97 & $59.2 / 57.3$ & 0.43 & $98.5 / 95.0$ & 0.34 \\
\hline \multicolumn{13}{|c|}{ Surgical margin } \\
\hline$(+) /(-)$ & $75.0 / 79.3$ & 0.95 & $50.0 / 50.8$ & 0.84 & $75.0 / 81.3$ & 0.75 & $100.0 / 88.5$ & 0.53 & $66.7 / 58.2$ & 0.59 & $100.0 / 97.6$ & 0.77 \\
\hline$\leq 1 />1 \mathrm{~mm}$ & $70.7 / 80.5$ & 0.51 & $46.3 / 49.6$ & 0.83 & $76.2 / 81.6$ & 0.64 & 100.0/87.1 & 0.20 & $54.8 / 57.8$ & 0.79 & $100.0 / 97.3$ & 0.55 \\
\hline$\leq 2 />2 \mathrm{~mm}$ & $81.0 / 78.2$ & 0.76 & $45.7 / 50.8$ & 0.93 & $84.8 / 79.5$ & 0.68 & $100.0 / 85.5$ & 0.08 & $50.5 / 60.2$ & 0.66 & $100.0 / 96.9$ & 0.42 \\
\hline \multicolumn{13}{|l|}{ Stage } \\
\hline $\mathrm{I} / \mathrm{II}$ & $90.6 / 71.1$ & 0.08 & $61.1 / 43.0$ & 0.09 & $93.0 / 72.5$ & $0.02^{\mathrm{a}}$ & $92.1 / 86.4$ & 0.49 & $69.0 / 50.5$ & $0.05^{\mathrm{a}}$ & $100.0 / 95.8$ & 0.20 \\
\hline \multicolumn{13}{|l|}{ BCLC stage } \\
\hline 0 & 83.8 & 0.49 & 58.0 & 0.64 & 83.8 & 0.56 & 100.0 & 0.15 & 58.0 & 0.75 & 100.0 & 0.60 \\
\hline A & 85.5 & & 43.6 & & 87.6 & & 83.1 & & 51.1 & & 97.7 & \\
\hline $\mathrm{B}$ & 70.0 & & 50.6 & & 73.2 & & 90.0 & & 64.4 & & 95.5 & \\
\hline
\end{tabular}

${ }^{\mathrm{a}} \mathrm{P}<0.05$. M, male; F, female; pT, pathological T stage; VI, vascular invasion; BCLC, Barcelona-Clinic Liver Cancer.

Table III. Prognostic factors affecting clinical outcomes in the multivariate analysis.

\begin{tabular}{lcccc}
\hline Factors & B & HR & $95 \%$ CI & P-value \\
\hline $\begin{array}{l}\text { Gender } \\
\quad \text { Male (ref.) }\end{array}$ & & 1 & & \\
$\quad$ Female & -1.10 & 0.33 & $(0.07-1.53)$ & \\
$\quad$ Vascular invasion & & & & \\
$\quad$ No (ref.) & & 1 & & 0.15 \\
$\quad$ Yes & 1.60 & 4.96 & $(1.55-15.90)$ & \\
Surgical margin (mm) & & & & 0.17 \\
$\quad$ 1 (ref.) & & 1 & & \\
$>1$ & -0.82 & 0.43 & $(0.13-1.45)$ & \\
BCLC stage & & & & 0.62 \\
0 (ref.) & & 1 & & \\
A & 0.23 & 1.26 & $(0.34-4.55)$ & \\
B & 0.60 & 1.83 & $(0.51-6.49)$ & \\
\hline
\end{tabular}

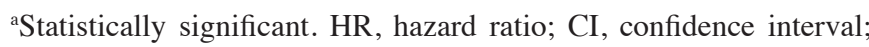
BCLC, Barcelona-Clinic Liver Cancer.

(range, 17.7-3.23 months). A total of 34 patients (38.2\%) had recurrent cancer; amongst these patients, initial tumor recurrence was confined to the original segment of the liver in
Table IV. Vascular invasion associated with intrahepatic recurrence.

\begin{tabular}{lcc}
\hline \multirow{2}{*}{$\begin{array}{l}\text { Intrahepatic } \\
\text { recurrence }\end{array}$} & \multicolumn{2}{c}{ Vascular invasion } \\
\cline { 2 - 3 } & No (\%) & Yes (\%) \\
\hline No & $39(78.0)$ & $22(56.4)$ \\
Yes & $11(22.0)$ & $17(43.6)$ \\
\hline $\mathrm{P}=0.03$. & & \\
\hline
\end{tabular}

4 patients $(4.5 \%)$ and was detected in different segments of the liver in 23 patients $(25.8 \%)$. In addition, there were 5 patients in both original and different segments of the liver. Two patients presented with distant treatment failure. The 3-year overall survival (OS) rate was $71 \%$. The univariate and Kaplan-Meier analyses indicated that vascular invasion was significantly associated with OS (Table II and Fig. 1). The 3-year OS rates in patients with and those without vascular invasion were 62.1 and $92.8 \%$, respectively $(\mathrm{P}<0.003)$. Based on the multivariate analysis, postoperative pathological vascular invasion (hazard ratio $=4.96 ; 95 \%$ confidence interval: $1.55-15.9)$ remained an independent predictor of adverse long-term outcome (Table III). Furthermore, vascular invasion was found to be significantly associated with intrahepatic recurrence (Table IV). 


\section{Discussion}

The outcome of hepatic resection for HCC has improved significantly due to the advances in surgical techniques and perioperative care. Partial hepatectomy for selected patients with HCC may now be performed with low operative morbidity and mortality rates $(10,11)$. However, long-term survival remains unsatisfactory due to the high incidence of recurrence, particularly in the residual liver (6). The intrahepatic recurrence rate following resection of HCC may be as high as $74 \%$ at 5 years (12). Another study also reported that recurrent disease accounted for $60 \%$ of late deaths following partial hepatectomy (13). In the present study, 34 patients developed recurrent cancer, within the original liver segment $(10.0 \%)$ or in other segments of the liver (31.5\%), which suggested that the majority of the recurrent tumors following hepatectomy originate from intrahepatic metastasis.

Patient and tumor characteristics and surgical variables affect recurrence following surgical resection for HCC. Vascular invasion is a significant factor affecting survival in HCC $(10,14)$. Pawlik et al $(10)$ reported that patients with vascular invasion exhibited a significantly shorter median survival compared to those without evidence of vascular invasion. Shah et al (14) observed that pathological vascular invasion was associated with a higher risk of early recurrence following liver resection. In the present study, we obtained similar results. The 3-year OS was significantly different between patients with pathologically proven vascular invasion and those without vascular invasion. Furthermore, recurrence in the majority of the patients was manifested as intrahepatic metastasis. Synchronous microscopic vascular invasion was associated with an increased risk of recurrence, even in patients with early-stage disease.

The improved survival following hepatectomy for HCC is mainly attributable to the prevention of recurrence. The incidence of intrahepatic recurrence may be as high as $74 \%$, suggesting a role for adjuvant therapy following hepatectomy (15). Aggressive treatment with a multimodality approach was suggested for patients with intrahepatic recurrence following curative resection and the survival of patients who underwent additional therapy was significantly improved (14). A number of adjuvant therapeutic options are currently available, including repeat hepatectomy, liver transplantation, radiofrequency ablation, TACE, hepatic arterial radioembolization, or radiation (stereotactic body radiation therapy and proton therapy) (15-19). In addition, antiangiogenic therapy, particularly sorafenib, has become the standard of care for patients with advanced HCC (20). Sorafenib has been found to suppress the development of postsurgical intrahepatic recurrence and prolong survival in an orthotopic mouse model (21). Those results strongly suggest that, by effectively reducing postoperative recurrence, adjuvant therapy has a potential application in early-stage HCC. However, further trials are required to compare the efficacy of these therapies and fully assess the benefit in selected patients at high risk for intrahepatic metastasis.

There remains controversy regarding the adequate surgical margins for recurrence. Poon et al (6), reported that resection margins ( $\leq 1$ vs. $>1 \mathrm{~cm})$ did not exert a significant effect on survival following recurrence. However, other studies reported that close surgical margins ( $\leq 5$ or $\leq 1 \mathrm{~mm}$ ) were correlated with a higher intrahepatic recurrence rate $(13,22)$. In the present study, surgical margins did not appear to affect survival. The possible reasons for the lack of a statistically significant difference may be that tumor recurrence mainly occurred in different segments of the liver. Efficient adjuvant treatment remains crucial in improving survival.

In conclusion, vascular invasion is an independent factor affecting survival in early HCC patients. The abovementioned data emphasize the need for effective adjuvant therapy in selected high-risk patients with early HCC. Further studies are required to identify the optimal strategy in the prevention and management of intrahepatic recurrences to further improve the prognosis of patients with early HCC.

\section{Acknowledgements}

This study was supported by the Buddhist Dalin Tzu Chi General Hospital [grant no. DTCRD101(2)-I-18].

\section{References}

1. Jemal A, Siegel R, Ward E, Hao Y, Xu J and Thun MJ: Cancer statistics, 2009. CA Cancer J Clin 59: 225-249, 2009.

2. Hwang S, Moon DB and Lee SG: Liver transplantation and conventional surgery for advanced hepatocellular carcinoma. Transpl Int 23: 723-727, 2010.

3. Hsieh CH, Liu CY, Shueng PW, et al: Comparison of coplanar and noncoplanar intensity-modulated radiation therapy and helical tomotherapy for hepatocellular carcinoma. Radiat Oncol 5: 40, 2010.

4. Fan ST, Lo CM, Liu CL, et al: Hepatectomy for hepatocellular carcinoma: toward zero hospital deaths. Ann Surg 229: 322-330, 1999.

5. Huang ZY, Liang BY, Xiong M, et al: Long-term outcomes of repeat hepatic resection in patients with recurrent hepatocellular carcinoma and analysis of recurrent types and their prognosis: a single-center experience in China. Ann Surg Oncol 19: 2515-2525, 2012

6. Poon RT, Fan ST, Lo CM, Liu CL and Wong J: Intrahepatic recurrence after curative resection of hepatocellular carcinoma: long-term results of treatment and prognostic factors. Ann Surg 229: 216-222, 1999.

7. Arnaoutakis DJ, Mavros MN, Shen F, et al: Recurrence patterns and prognostic factors in patients with hepatocellular carcinoma in noncirrhotic liver: a multi-institutional analysis. Ann Surg Oncol 21: 147-154, 2014.

8. Kornberg A, Kupper B, Tannapfel A, et al: Long-term survival after recurrent hepatocellular carcinoma in liver transplant patients: clinical patterns and outcome variables. Eur J Surg Oncol 36: 275-280, 2010.

9. Sumie S, Nakashima O, Okuda K, et al: The significance of classifying microvascular invasion in patients with hepatocellular carcinoma. Ann Surg Oncol 21: 1002-1009, 2014.

10. Pawlik TM, Poon RT, Abdalla EK, et al: Critical appraisal of the clinical and pathologic predictors of survival after resection of large hepatocellular carcinoma. Arch Surg 140: 450-458, 2005.

11. Chok KS, Ng KK, Poon RT, Lo CM and Fan ST: Impact of postoperative complications on long-term outcome of curative resection for hepatocellular carcinoma. Br J Surg 96: 81-87, 2009.

12. Yamamoto J, Kosuge T, Takayama T, Shimada K, Yamasaki S, Ozaki H, Yamaguchi N and Makuuchi M: Recurrence of hepatocellular carcinoma after surgery. Br J Surg 83: 1219-1222, 1996.

13. Nagasue N, Uchida M, Makino Y, et al: Incidence and factors associated with intrahepatic recurrence following resection of hepatocellular carcinoma. Gastroenterology 105: 488-494, 1993.

14. Shah SA, Cleary SP, Wei AC, et al: Recurrence after liver resection for hepatocellular carcinoma: risk factors, treatment, and outcomes. Surgery 141: 330-339, 2007.

15. Cha C, Fong Y, Jarnagin WR, Blumgart LH and DeMatteo RP: Predictors and patterns of recurrence after resection of hepatocellular carcinoma. J Am Coll Surg 197: 753-758, 2003. 
16. Wu L, Hu A, Tam N, et al: Salvage liver transplantation for patients with recurrent hepatocellular carcinoma after curative resection. PLoS One 7: e41820, 2012.

17. Lee DH, Lee JM, Lee JY, Kim SH, Han JK and Choi BI: Radiofrequency ablation for intrahepatic recurrent hepatocellular carcinoma: long-term results and prognostic factors in 168 patients with cirrhosis. Cardiovasc Intervent Radiol 37: 705-715, 2014

18. Huang WY, Jen YM, Lee MS, et al: Stereotactic body radiation therapy in recurrent hepatocellular carcinoma. Int J Radiat Oncol Biol Phys 84: 355-361, 2012.

19. Sugahara S, Nakayama H, Fukuda K, et al: Proton-beam therapy for hepatocellular carcinoma associated with portal vein tumor thrombosis. Strahlenther Onkol 185: 782-788, 2009.
20. Abou-Alfa GK, Schwartz L, Ricci S, et al: Phase II study of sorafenib in patients with advanced hepatocellular carcinoma. J Clin Oncol 24: 4293-4300, 2006

21. Feng YX, Wang T, Deng YZ, et al: Sorafenib suppresses postsurgical recurrence and metastasis of hepatocellular carcinoma in an orthotopic mouse model. Hepatology 53: 483-492, 2011.

22. Shah SA, Greig PD, Gallinger S, et al: Factors associated with early recurrence after resection for hepatocellular carcinoma and outcomes. J Am Coll Surg 202: 275-283, 2006. 